\title{
Papillary Muscle Dysfunction Due to Coronary Slow-Flow Phenomenon Presenting with Acute Mitral Regurgitation and Unilateral Pulmonary Edema
}

\author{
Jose Chacko, Gagan Brar, Bhargav Mundlapudi, Pradeep Kumar \\ Department of Critical Care Medicine, Narayana Multispecialty Hospital, Bengaluru, Karnataka, India
}

Abstract

Cardiogenic pulmonary edema usually presents with characteristic clinical features and bilateral infiltrates on the chest radiograph. Rarely, pulmonary edema may manifest unilaterally, leading to a mistaken diagnosis of a primary lung pathology. We present a 30-year-old man who developed acute coronary syndrome following an overdose of alprazolam. He developed breathlessness with unilateral infiltrates on the chest radiograph. Echocardiography revealed regional wall motion abnormalities related to underlying ischemia and acute mitral regurgitation with an eccentric jet. Besides, he had significant impairment of left ventricular systolic function. His coronary angiogram revealed a slow-flow phenomenon in the right coronary and left anterior descending artery territories. Ischemia-related dysfunction of the posterolateral papillary muscle probably led to a floppy posterior mitral leaflet and an eccentrically directed regurgitant jet, leading to unilateral pulmonary edema. He was commenced on dual antiplatelet therapy, heparin infusion, atorvastatin, frusemide, and ramipril, following which he showed gradual clinical improvement along with resolution of the radiological infiltrates. His left ventricular function improved, and the mitral valve function normalized on echocardiography within a week.

Keywords: Acute coronary syndrome, coronary slow-flow phenomenon, papillary muscle dysfunction, unilateral pulmonary edema

\section{INTRODUCTION}

Acute cardiogenic pulmonary edema usually presents with bilateral infiltrates on the chest radiograph described as a "butterfly shadow."'[1] Unilateral pulmonary edema (UPE) of cardiac origin is relatively rare and often misdiagnosed as primary respiratory disease leading to delay in the initiation of appropriate therapy. ${ }^{[2]}$ We report a young man who presented with breathlessness and unilateral lung infiltrates following an overdose with alprazolam and review the literature on UPE of cardiac origin.

\section{Case Report}

A 30-year-old man presented to our emergency department with breathlessness following an overdose with alprazolam. He had been evaluated earlier at another hospital, where he was found to be hypotensive. Ischemic changes were noted on electrocardiography (ECG); he was cardioverted as he was found to be in atrial fibrillation. Following this, he was transferred to our hospital.

\begin{tabular}{|l|l|}
\hline \multicolumn{3}{c|}{ Access this article online } \\
\hline Quick Response Code: & Website: \\
& www.ijccm.org \\
& \\
\end{tabular}

On initial evaluation in our emergency department, he had mild dyspnea, with a respiratory rate of $32 / \mathrm{min}$ and oxygen saturation of $88 \%$ on room air. His heart rate was regular at $105 / \mathrm{min}$ with a blood pressure of $86 / 52 \mathrm{~mm} \mathrm{Hg}$. Systemic examination was otherwise unremarkable. He was administered supplemental oxygen, commenced on an infusion of noradrenaline, and transferred to the intensive care unit. His ECG performed earlier at the referring hospital, showed atrial fibrillation with ST-segment elevation in leads II and III, and ST-segment depression in leads V3-V4 [Figure 1]. A chest radiograph revealed diffuse infiltrates confined to the right lung fields, suggestive of UPE [Figure 2]. On transthoracic echocardiography, he had significant hypokinesia of the basal,

Address for correspondence: Dr. Jose Chacko, Department of Critical Care Medicine, Narayana Multispecialty Hospital, Bengaluru, Karnataka, India. E-mail: chackojose@gmail.com

This is an open access journal, and articles are distributed under the terms of the Creative Commons Attribution-NonCommercial-ShareAlike 4.0 License, which allows others to remix, tweak, and build upon the work non-commercially, as long as appropriate credit is given and the new creations are licensed under the identical terms.

For reprints contact: reprints@medknow.com

How to cite this article: Chacko J, Brar G, Mundlapudi B, Kumar P. Papillary muscle dysfunction due to coronary slow-flow phenomenon presenting with acute mitral regurgitation and unilateral pulmonary edema. Indian J Crit Care Med 2018;22:806-8. 
inferoseptal, and basal posterior walls with impaired systolic function. Mitral regurgitation (MR) of moderate degree was also observed, with an eccentrically directed regurgitant jet [Figure 3]. The creatine kinase level was $23.3 \mathrm{ng} / \mathrm{ml}$ (normal range: $0-4.3 \mathrm{ng} / \mathrm{ml}$ ) and the troponin-I level, $5.66 \mathrm{ng} / \mathrm{ml}$ (normal range: $0-0.02 \mathrm{ng} / \mathrm{ml}$ ). Acute coronary syndrome with acute MR was suspected, and a coronary angiogram was carried out.

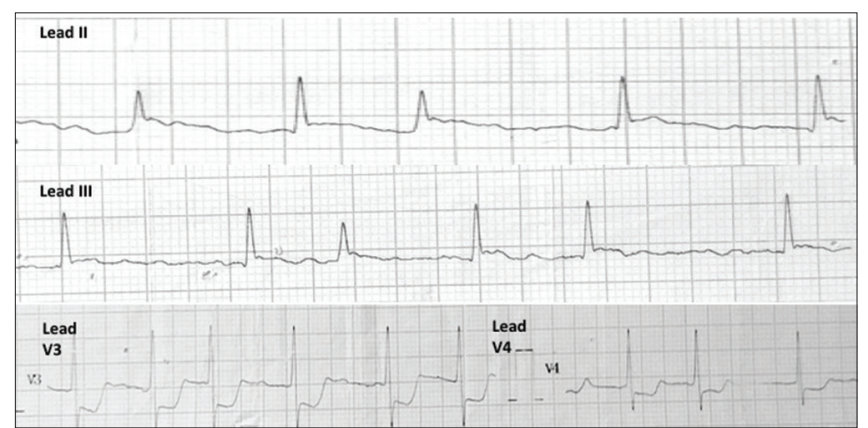

Figure 1: Electrocardiography showing ST-segment elevation in leads II and III and ST-segment depression in leads V3-V4

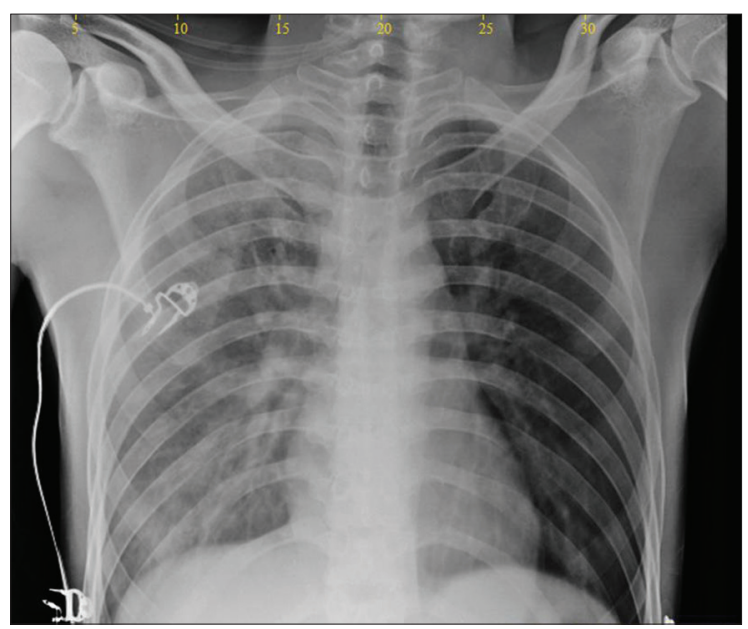

Figure 2: Chest radiograph showing unilateral pulmonary edema on the right side

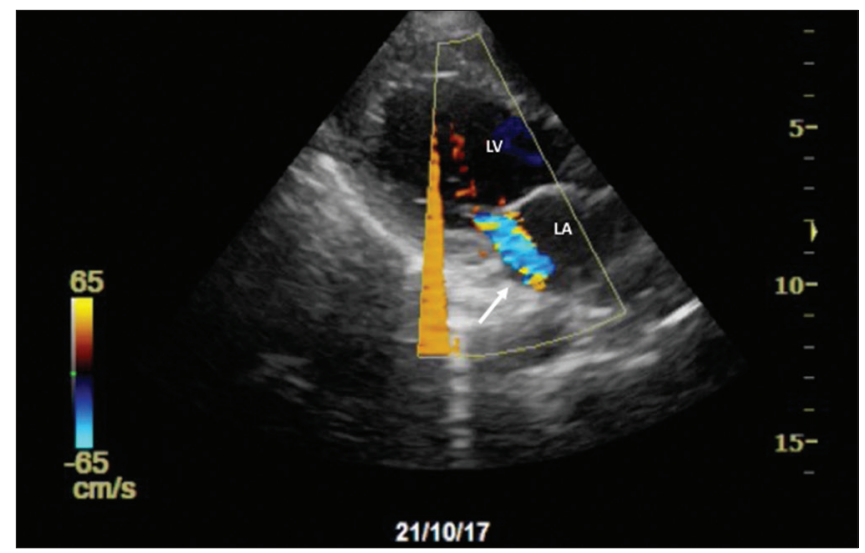

Figure 3: Transthoracic echocardiogram. The arrow points to the eccentrically directed mitral regurgitant jet. LA: Left atrium; LV: Left ventricle
Normal flows were noted in the left main and circumflex arteries. The right coronary artery was dominant with no stenotic lesion; however, a slow-flow pattern was observed. Slow flow was also observed in the left anterior descending artery. The flow rates were estimated using the corrected thrombolysis in myocardial infarction frame count as described previously by Gibson et al.$^{[3]}$

Based on these findings, a diagnosis of the acute coronary syndrome with acute MR due to papillary muscle dysfunction was considered, and he was commenced on aspirin, clopidogrel, heparin infusion, ramipril, and frusemide. His breathlessness gradually improved and the infiltrates on the chest radiograph disappeared within a week. A repeat echocardiogram at this time showed improved left ventricular systolic function and the MR was no longer evident.

\section{Discussion}

UPE may arise from cardiac or noncardiac causes. While bilateral pulmonary edema has typical clinical and radiographic features, UPE is relatively uncommon. ${ }^{[4]}$ The presence of unilateral infiltrates on the chest radiograph may lead to a misdiagnosis of pneumonia and delay in the initiation of appropriate therapy. ${ }^{[2]}$ Several noncardiac causes have been reported, including rapid lung reexpansion, ${ }^{[5]}$ lying in the lateral position for prolonged periods, ${ }^{[6]}$ fluid overload, ${ }^{[7]}$ and following endobronchial intubation. ${ }^{[8]}$ In a retrospective analysis of 869 patients with cardiogenic pulmonary edema, UPE was observed in $18(2.1 \%)$ of patients and was associated with higher mortality compared to bilateral pulmonary edema. Severe MR was noted in all patients who presented with cardiogenic UPE in this study. ${ }^{[4]}$

Our patient presented with features of acute coronary syndrome following an overdose of alprazolam. He had radiographic features of right-sided UPE. His initial ECG showed ST-segment elevation in the anterolateral leads. On transthoracic echocardiography, he had significant hypokinesia of the basal, inferoseptal, and basal posterior walls with impaired systolic function. Besides, he had a moderate degree of MR with an eccentric jet, resulting in right-sided pulmonary edema. Ischemia-related dysfunction of the posteromedial papillary muscle probably resulted in a floppy posterior mitral leaflet; the consequent eccentric regurgitant jet was directed toward the right pulmonary vein leading to right-sided pulmonary edema in our patient.

UPE due to MR usually occurs on the right side, ${ }^{[4]}$ as seen in our patient. The anatomical configuration of the mitral valve tends to direct a regurgitant jet toward the right pulmonary vein, ${ }^{[9]}$ especially to the right superior pulmonary vein, leading to a predilection for edema formation in the right upper lobe. ${ }^{[10]}$ Furthermore, the pulmonary capillary wedge pressure may be higher in the right upper lobe, ${ }^{[10]}$ predisposing to the localization of edema to this area of the lung.

Branches of the coronary arteries traverse the whole thickness of the myocardium before they reach the subendocardial layer 
to perfuse the papillary muscles, making them susceptible to ischemic insult. ${ }^{[11]}$ The posteromedial papillary muscle is more vulnerable to ischemia-related dysfunction because of a single arterial supply by the posterior descending or the circumflex artery. ${ }^{[11]}$ Papillary muscle dysfunction due to ischemia may be episodic and reversible. ${ }^{[12]}$ Our patient probably developed transient impairment of left ventricular function and MR related to reversible papillary muscle dysfunction, which recovered completely within a few days.

The coronary angiogram in our patient did not reveal any stenotic lesion; however, a slow-flow phenomenon was observed in the right coronary and the left anterior descending arteries. The coronary slow flow phenomenon (CSFP) is a well-recognized cause of acute coronary syndromes, characterized by delayed opacification of the distal coronary arteries in the absence of epicardial occlusive disease. ${ }^{[13,14]}$ CSFP has been postulated to be due to underlying small vessel disease, endothelial dysfunction, inflammation, or due to anatomical factors, including tortuosity and branching. ${ }^{[14]}$ Our patient had a right dominant coronary circulation, wherein the posterior descending branch arises from the right coronary artery. A CSFP involving the right coronary artery may have led to compromised flow in the posterior descending artery, resulting in ischemia and dysfunction of the posteromedial papillary muscle.

Our patient presented with several unique features. He presented following an overdose with alprazolam. Subsequently, he developed acute coronary syndrome related to CSFP and developed UPE. He had ECG and echocardiographic evidence of acute coronary syndrome involving multiple territories, with impaired left ventricular function. The UPE was related to an eccentric MR jet due to papillary muscle dysfunction.

\section{ConcLusion}

UPE is relatively rare and may occur due to MR with an eccentric jet. Acute MR with an eccentric jet may develop due to papillary muscle dysfunction, induced by CSFP. It is important to distinguish cardiogenic UPE from radiographic infiltrates arising from a primary lung pathology and initiate appropriate therapy without delay.

\section{Declaration of patient consent}

The authors certify that they have obtained all appropriate patient consent forms. In the form the patient(s) has/have given his/her/their consent for his/her/their images and other clinical information to be reported in the journal. The patients understand that their names and initials will not be published and due efforts will be made to conceal their identity, but anonymity cannot be guaranteed.

\section{Financial support and sponsorship \\ Nil.}

\section{Conflicts of interest}

There are no conflicts of interest.

\section{References}

1. Gropper MA, Wiener-Kronish JP, Hashimoto S. Acute cardiogenic pulmonary edema. Clin Chest Med 1994;15:501-15.

2. Choi HS, Choi H, Han S, Kim HS, Lee C, Kim YY, et al. Pulmonary edema during pregnancy: Unilateral presentation is not rare. Circ $\mathrm{J}$ 2002;66:623-6.

3. Gibson CM, Cannon CP, Daley WL, Dodge JT Jr., Alexander B Jr., Marble SJ, et al. TIMI frame count: A quantitative method of assessing coronary artery flow. Circulation 1996;93:879-88.

4. Attias D, Mansencal N, Auvert B, Vieillard-Baron A, Delos A, Lacombe $\mathrm{P}$, et al. Prevalence, characteristics, and outcomes of patients presenting with cardiogenic unilateral pulmonary edema. Circulation 2010;122:1109-15.

5. Murat A, Arslan A, Balci AE. Re-expansion pulmonary edema. Acta Radiol 2004;45:431-3.

6. Leeming BW. Gravitational edema of the lungs observed during assisted respiration. Chest 1973;64:719-22.

7. Balogun SA, Balogun RA. Acute unilateral pulmonary edema from dietary salt and water load: A case report and review of the literature. Conn Med 2001;65:653-6.

8. Kramer MR, Melzer E, Sprung CL. Unilateral pulmonary edema after intubation of the right mainstem bronchus. Crit Care Med 1989;17:472-4.

9. Miyatake K, Nimura Y, Sakakibara H, Kinoshita N, Okamoto M, Nagata S, et al. Localisation and direction of mitral regurgitant flow in mitral orifice studied with combined use of ultrasonic pulsed Doppler technique and two dimensional echocardiography. Br Heart J 1982;48:449-58.

10. Gurney JW, Goodman LR. Pulmonary edema localized in the right upper lobe accompanying mitral regurgitation. Radiology 1989;171:397-9.

11. Inoue T, Iemura J, Saga T. Successful surgical treatment of mitral regurgitation for complete rupture of the anterior papillary muscle after acute myocardial infarction. Jpn J Thorac Cardiovasc Surg 2003;51:565-8.

12. Le Feuvre C, Metzger JP, Lachurie ML, Georges JL, Baubion N, Vacheron A, et al. Treatment of severe mitral regurgitation caused by ischemic papillary muscle dysfunction: Indications for coronary angioplasty. Am Heart J 1992;123:860-5.

13. Beltrame JF, Limaye SB, Horowitz JD. The coronary slow flow phenomenon - A new coronary microvascular disorder. Cardiology 2002;97:197-202.

14. Wang X, Nie SP. The coronary slow flow phenomenon: Characteristics, mechanisms and implications. Cardiovasc Diagn Ther 2011;1:37-43. 